\title{
Primary Pancreatic Tuberculosis: A Rare Case Report
}

\author{
Konan Bharat, Chellappa Vijayakumar, TP Elamurugan*, Sudharsanan Sundaramurthi and Sadasivan Jagdish \\ Department of Surgery, Jawaharlal Institute of Postgraduate Medical Education and Research (JIPMER), India
}

Submission: May 17, 2019; Published: June 14, 2019

*Corresponding author: TP Elamurugan, Associate Professor, Department of Surgery, Jawaharlal Institute of Postgraduate Medical Education and Research (JIPMER), Puducherry, India

Abstract

Tuberculosis (TB) is a serious health problem worldwide. Pancreatic tuberculosis (PTB) is a rare occurrence seen in immunosuppressed individuals. It usually occurs in patients with miliary TB with pulmonary and extra-pulmonary involvement. Even though it is a relatively rare occurrence, it is said to be an Acquired Immune Deficiency Syndrome (AIDS) defining illness. Primary Pancreatic tuberculosis (PPTB) is the isolated involvement of pancreas by Mycobacterium tuberculosis in the absence of involvement of any other organ or previously identified TB. It has a reported incidence of only $0.46 \%$ among patients with TB.

We report a case of a 30-year-old gentleman, previously diagnosed to have been infected with Human Immunodeficiency Virus (HIV) and on antiretroviral therapy (ART), who presented with epigastric pain and high-grade fever for 4 weeks. Contrast Enhanced Computed Tomography (CECT) scan showed a bulky oedematous pancreas with a well-defined collection in the body. Ultrasound (USG) guided Fine Needle Aspiration (FNA) of the fluid revealed pus and the cultures grew M. tuberculosis. There were no other foci of TB in the patient. Anti-tubercular therapy (ATT) was initiated and resulted in gradual resolution of symptoms.

PPTB is often undiagnosed due to low index of suspicion and because its symptoms mimic more common pancreatic conditions. Since it is a treatable disease, accurate diagnosis is vital in order to initiate appropriate pharmacotherapy.

Keywords: Pancreatic tuberculosis; Pharmacotherapy; Tuberculosis; Anti-tubercular therapy; Treatable disease; Ultrasound; Extra-pulmonary TB; HIV-seropositive; Pancreas; Edematous pancreas; Peri-pancreatic inflammation; Neutrophils; Lymphocytes; Eosinophils; Monocytes

Abbreviations: TB: Tuberculosis; PPTB: Primary Pancreatic Tuberculosis; PTB: Pancreatic Tuberculosis; AIDS: Acquired Immune Deficiency Syndrome; ART: Antiretroviral Therapy; HIV: Immunodeficiency Virus; CECT: Contrast Enhanced Computed Tomography; USG: Ultrasound; FNA: Fine Needle Aspiration; ATT: Anti-Tubercular Therapy; HIV: Human Immunodeficiency Virus

\section{Introduction}

Tuberculosis (TB) is a worldwide public health problem, which has a diverse mode of presentation and the diagnosis is difficult and delayed in some rarer presentations [1]. In India, the incidence of TB in 2015 was 217 per lakh population and the mortality due to TB was 36 per lakh population. Primary Pancreatic tuberculosis (PPTB) is a rare entity among patients with extra-pulmonary TB [2]. Isolated pancreatic TB is predominantly observed in patients who reside in endemic TB zones, in areas of widespread TB dissemination such as a military setting and patients in developing countries who are immuno-compromised.

Majority of cases occur in endemic zones and in immunocompromised patients [2]. There are only a few reported cases of isolated tubercular involvement of pancreas [3]. TB is an extremely common opportunistic infection in Human Immunodeficiency Virus (HIV) positive patients and is considered to be an Acquired Immune Deficiency Syndrome (AIDS)-defining illness. Because of the virulence of TB, its symptoms tend to manifest at an early stage of HIV infection. The most overt feature of TB in HIV-sero positive patients is their substantially greater likelihood of having extra-pulmonary involvement and dissemination [4].

\section{Case Presentation}

A 30-year-old gentleman presented with complaints of upper abdominal pain, fever, weight loss and generalised malaise for onemonth duration. He was recently diagnosed to be HIV positive and was started on antiretroviral therapy (ART). He was not on any treatment for TB or had contact with patients with TB in the past. At admission, patient was conscious, oriented and his vitals were stable. The patient was averagely built, moderately nourished and afebrile at the time of examination. On examination tenderness was present in the epigastrium, with mild guarding. All hematological (Hb 12 gm/dl, Total Leukocyte counts - 6100 cells/cubic $\mathrm{mm}$, Differential Leukocyte count - Neutrophils - 74\%, Lymphocytes - 22\%, Eosinophils - 3\% and Monocytes -1\%) and biochemical investigations (blood sugar $92 \mathrm{mg} / \mathrm{dL}$, blood urea $10 \mathrm{mg} / \mathrm{dL}$ Amylase $251 \mathrm{IU} / \mathrm{L}$ ) were within normal reference ranges except for increased erythrocyte sedimentation rate (ESR) of $60 \mathrm{~mm}$ in 
the first hour. On further investigation his Chest X-ray was normal. Ultrasound (USG) suggested bulky edematous pancreas with peri-pancreatic inflammation, and evidence of collection $(5 \times 5 \mathrm{~cm})$ in the body of pancreas with moving echoes. Contrast Enhanced Computed Tomography (CECT) was done and it favoured acute pancreatitis with pancreatic abscess (Figure 1).

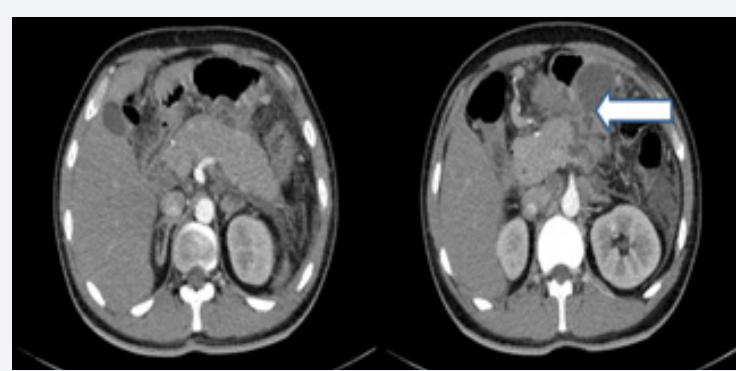

Figure 1: CECT Abdomen showing edematous pancreas and peripancreatic collection (white arrow).

CECT: Contrast Enhanced Computed Tomography

In the CECT, pancreas was bulky, edematous with surrounding fat stranding. Multiple lymph nodes were present in the peri-pancreatic region with the largest diameter of $8 \mathrm{~mm}$. Collection was present in the body of pancreas $(6 \times 5 \mathrm{~cm})$ extending into the lesser sac. Bowel was appearing normal in calibre.

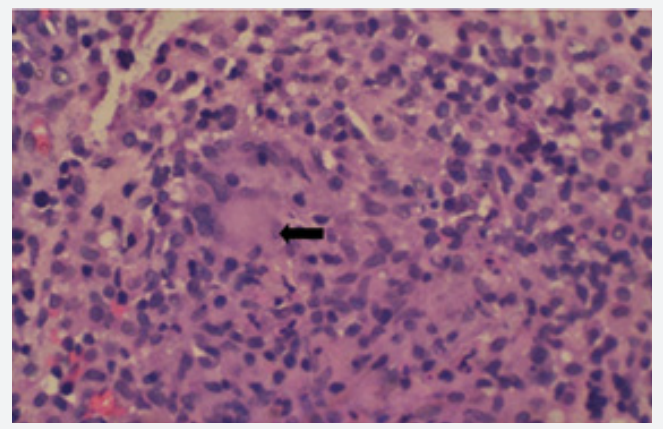

Figure 2: Histopathological picture (H\&E stain, 400X magnification) is showing epithelioid cell granuloma with characteristic Langhans Giant cells (black arrow) surrounded by lymphocytes and plasma cells.

H\&E: Haemotoxylin and Eosin

Patient was started on antibiotics based on our routine institute protocol (Ceftriaxone, Amikacin and Metronidazole). Therapeutic aspiration from the abscess was done. Thick pus (50 ml) was aspirated and sent for bacterial culture. In view of his immunocompromised state Ziehl-Neelson staining and Acid-Fast Bacilli (AFB) culture was done. The pus was found to be positive for AFB (Figure 2).

Patient was started on Anti-tubercular therapy (ATT) subsequently. Following ATT, patient improved symptomatically and was discharged after two weeks.

\section{Discussion}

India accounts for one-fourth of the global incidence of TB, with nearly 2.8 million incident cases in the year 2015. Nearly, 15$20 \%$ of all TB cases occur in extra-pulmonary sites. Among the ex- tra-pulmonary sites, the most common are TB lymph nodes (35\%) followed by pleural TB (20\%) and TB in bones and joints $(10 \%)$ [3]. Pancreatic tuberculosis (PT) is rare and can occur in the absence of evidence of TB elsewhere in the body [4]. PT typically presents in patients who reside in endemic tuberculous zones, sporadically in no-risk healthy patients, and in patients who are immuno-compromised [5].

A wide spectrum of symptoms has been reported. It ranges from abdominal pain, constitutional symptoms such as weight loss, night sweats and anorexia, fever, obstructive jaundice, to massive gastro-intestinal bleeding and iron deficiency anemia and pancreatic abscess [6].

The most common route of spread of TB to the solid organs of abdomen is the haematogenous route and less commonly the lymphatic route. In abdominal TB, ileocaecal region is most commonly affected; solid organs like kidney, spleen, and liver get involved with TB much more commonly than the pancreas. PT can occur as a result of haematogenous or lymphatic dissemination or direct spread from adjacent organs. The uncommon occurrence of PT is probably due to the presence of pancreatic enzymes, which interfere with the seeding of Mycobacterium tuberculosis. Pancreatic secretions have been reported to have anti-tubercular effect in vitro, suggesting a potential protective mechanism for the rare pancreatic involvement with TB [7].

Extra-pulmonary TB is not unusual in HIV seropositive patients from countries with a high prevalence of TB. However, in such patients isolated TB of the pancreas is unusual [8]. The most important differential diagnosis includes pancreatic malignancy. PT may present with a wide range of imaging findings. It is difficult to diagnose PT on imaging studies as they may present with masses, cystic lesions or abscesses and mass lesions in most of the cases mimic pancreatic carcinoma.

In AIDS cases, tuberculous pancreatic abscesses are most common, accounting for $70 \%$ of cases. If the diagnosis is delayed, pancreatic TB can be fatal and the disease has a $10.8 \%$ mortality rate which is comparable to the mortality rate of $9.1 \%$ in immunocompetent patients [9]. Endoscopic USG or image guided needle aspiration is the recommended diagnostic technique. Although the prognosis is good with ATT, it could be fatal without correct diagnosis and treatment [10].

Therefore, it is important to obtain tissue for appropriate histological and microbiological analyses, highlighting the need for laparoscopy or laparotomy in most published cases of PT.

\section{Conclusion}

High index of suspicion must be maintained for PT in immunocompromised patients (especially HIV patients) who present with short duration of non-specific symptoms of pancreatic disease. Imaging is helpful but tissue diagnosis is the cornerstone for management. ATT is highly effective for treatment. As it is a rare entity, it cannot be recommended but suggested that PT should be considered in cases with a large space occupying lesions associat- 
ed with necrotic peripancreatic lymph nodes and constitutional symptoms.

\section{References}

1. Shahrokh S, Miri MB, Safari MT, Houshang A, Alizadeh AHM (2015) Pancreatic Tuberculosis: An Overview. J Pancreas 16: 232-238.

2. Dang S, Atiq M, Saccente M, Olden KW, Aduli F (2009) Isolated Tuberculosis of the Pancreas: A Case Report. J Pancreas 10(1): 64-66.

3. Rana SS, Bhasin DK, Rao C, Singh K (2010) Isolated Pancreatic Tuberculosis Mimicking Focal Pancreatitis and Causing Segmental Portal Hypertension. J Pancreas 11(4): 393-395.

4. Falkowski AL, Graber J, Haack HG, Tarr PE, Rasch H (2013) Isolated pancreatic tuberculosis: A case report and radiological comparison with cystic pancreatic lesions. J Radiol Case Rep 7(1): 1-11.

5. Meesiri S (2012) Pancreatic tuberculosis with acquired immunodeficiency syndrome: A case report and systematic review. World J Gastroenterol 18(7): 720-726.

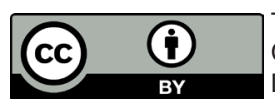

This work is licensed under Creative Commons Attribution 4.0 License DOI: 10.19080/ARGH.2019.13.555858
6. Yang YJ, Li YX, Liu XQ, Yang M, Liu K (2014) Pancreatic tuberculosis mimicking pancreatic carcinoma during anti-tuberculosis therapy: A case report. World J Clin Cases 2(5): 167-169.

7. Kaushik N, Schoedel K, McGrath K (2006) Isolated pancreatic tuberculosis diagnosed by endoscopic ultrasound-guided fine needle aspiration: A case report. J Pancreas 7(2): 205-210.

8. Porter AE (1917) The Bacteriolytic Action of Gland Extracts on Tubercle Bacilli. J Hyg (Lond) 16(1): 55-65.

9. Franco-Paredes C, Leonard M, Jurado R, Blumberg HM, Smith RM (2002) Tuberculosis of the pancreas: report of two cases and review of the literature. Am J Med Sci 323(1): 54-58.

10. Loya AC, Prayaga AK, Sundaram C, Shantharam V, Kumar A (2005) Cytologic diagnosis of pancreatic tuberculosis in immunocompetent and immunocompromised patients: a report of 2 cases. Acta Cytol 49(1): 97-100.

\section{Your next submission with JuniperPublishers will reach you the below assets}

- Quality Editorial service

- Swift Peer Review

- Reprints availability

- E-prints Service

- Manuscript Podcast for convenient understanding

- Global attainment for your research

- Manuscript accessibility in different formats

( Pdf, E-pub, Full Text, audio)

- Unceasing customer service

Track the below URL for one-step submission

https://juniperpublishers.com/online-submission.php 